\title{
Crystal structure of methyl-2-hydroxy-2-phenyl-3-(4-bromophenyl)-3- phenylamino-propanoate, $\mathrm{C}_{22} \mathrm{H}_{20} \mathrm{BrNO}_{3}$
}

\author{
L. Malpezzi* \\ Politecnico di Milano. Dipartimento di Chimica, Materiali e Ingegneria Chimica "G. Natta", Via Mancinelli 7, I-20131 Milano, Italy
}

Received March 12, 2004, accepted and available on-line May 4, 2004; CCDC no. 1267/1273

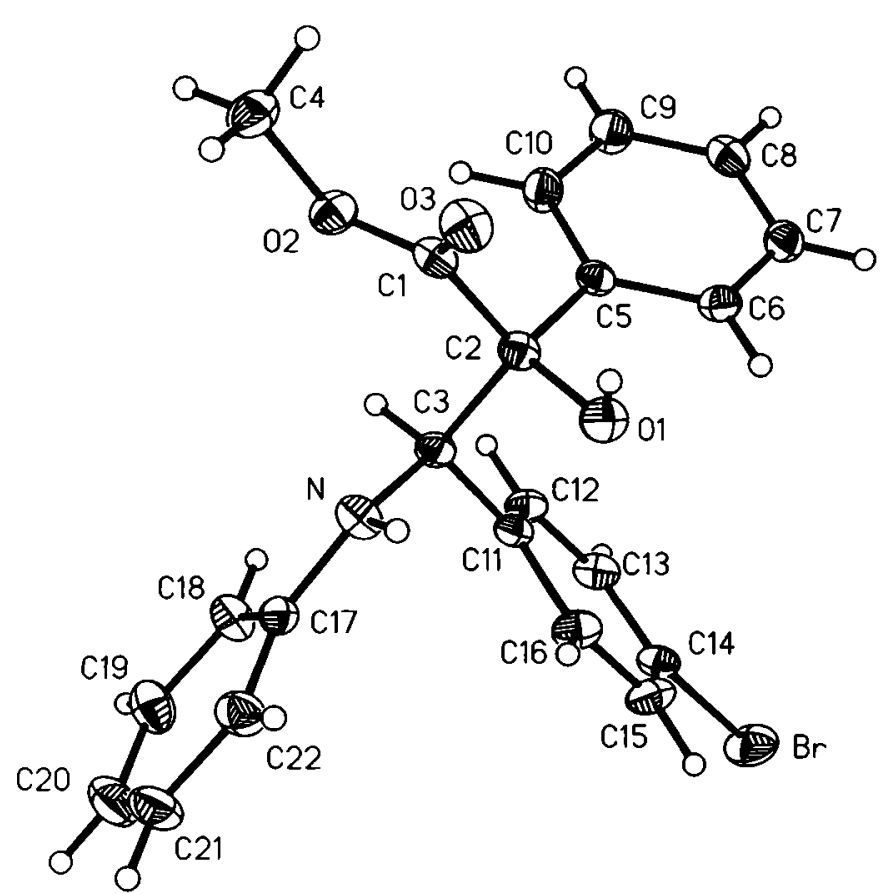

isomer of the $\beta$-amino- $\alpha$-hydroxyester and shows a torsion angle $\mathrm{O} 1-\mathrm{C} 2-\mathrm{C} 3-\mathrm{N}$ of $60.6^{\circ}$. The environment of the amino $\mathrm{N}$ atom is not planar. The $\mathrm{N}$ atom is lying $0.23 \AA$ out of plane of its three substituents. In the crystal the molecules are stacked in columns parallel to $b$ axis. Weak intra- and inter-molecular hydrogen bonds are present.

Table 1. Data collection and handling.

\begin{tabular}{ll}
\hline Crystal: & colorless needle, size $0.1 \times 0.1 \times 0.6 \mathrm{~mm}$ \\
Wavelength: & Cu $K_{\alpha}$ radiation $(1.54178 \AA)$ \\
$\mu:$ & $30.26 \mathrm{~cm}^{-1}$ \\
Diffractometer, scan mode: & Bruker P4, $\theta / 2 \theta$ \\
$2 \theta_{\text {max }}:$ & $113.98^{\circ}$ \\
$\left.N(h k l)_{\text {measured }}, N(h k l)\right)_{\text {unique: }}:$ & 2753,2650 \\
Criterion for $I_{\text {obs, }} N(h k l)_{\text {gl }}:$ & $I_{\text {obs }}>2 \sigma\left(I_{\text {obs }}\right), 2130$ \\
$N(\text { param })_{\text {refined: }}$ & 259 \\
Programs: & SIR97 [2], SHELXL-97 [3], \\
& SHELXTL [4]
\end{tabular}

Abstract

$\mathrm{C}_{22} \mathrm{H}_{20} \mathrm{BrNO}_{3}$, monoclinic, $P 12_{1} / c 1$ (no. 14),

$a=17.643(1) \AA, b=5.935(1) \AA, c=18.782(1) \AA$,

$\beta=90.13(1)^{\circ}, V=1966.7 \AA^{3}, Z=4$,

$R_{\mathrm{gt}}(F)=0.041, w R_{\text {ref }}\left(F^{2}\right)=0.117, T=293 \mathrm{~K}$.

\section{Source of material}

The compound was prepared by a condensation of three components (methyl phenylglyoxylate, aniline and aromatic aldehyde) promoted by $\mathrm{TiCl}_{3}$ /pyridine in anhydrous THF at room temperature. The details of the stereoselective synthesis and the proposed mechanism of reaction have already been described [1]. Colorless crystals were obtained by crystallization from a diethyl ether/hexane solution.

\section{Experimental details}

The hydrogen atoms being chemically relevant were refined isotropically, whereas the rest was treated as riding model.

\section{Discussion}

The crystal structure of the title compound was determined in order to understand the stereochemical feature of the two asymmetric centres at $\mathrm{C} 2$ and $\mathrm{C} 3$. The molecule was found to be the syn

Table 2. Atomic coordinates and displacement parameters (in $\AA^{2}$ ).

\begin{tabular}{llllll}
\hline Atom & Site & $x$ & \multicolumn{1}{l}{$y$} & $z$ & $U_{\text {iso }}$ \\
\hline $\mathbf{H}(1 \mathrm{~N})$ & $4 e$ & $0.132(2)$ & $0.056(6)$ & $-0.272(2)$ & $0.04(1)$ \\
$\mathbf{H}(10)$ & $4 e$ & $0.230(3)$ & $0.297(8)$ & $-0.346(3)$ & $0.07(2)$ \\
$\mathbf{H}(3)$ & $4 e$ & $0.230(2)$ & $-0.267(5)$ & $-0.270(2)$ & $0.030(8)$ \\
$\mathbf{H}(41)$ & $4 e$ & 0.1768 & -0.4924 & -0.4744 & 0.076 \\
$\mathbf{H}(42)$ & $4 e$ & 0.2075 & -0.2716 & -0.5100 & 0.076 \\
$\mathbf{H}(43)$ & $4 e$ & 0.1268 & -0.2740 & -0.4753 & 0.076 \\
$\mathbf{H}(6)$ & $4 e$ & 0.3715 & 0.2925 & -0.2910 & 0.058 \\
$\mathbf{H}(7)$ & $4 e$ & 0.5007 & 0.2482 & -0.3007 & 0.065 \\
$\mathbf{H}(8)$ & $4 e$ & 0.5505 & -0.0764 & -0.3490 & 0.067 \\
$\mathbf{H}(9)$ & $4 e$ & 0.4707 & -0.3632 & -0.3826 & 0.071 \\
$\mathbf{H}(10)$ & $4 e$ & 0.3412 & -0.3231 & -0.3720 & 0.065 \\
$\mathbf{H}(12)$ & $4 e$ & 0.3363 & -0.2980 & -0.1865 & 0.054 \\
$\mathbf{H}(13)$ & $4 e$ & 0.4002 & -0.1826 & -0.0849 & 0.062 \\
$\mathbf{H}(15)$ & $4 e$ & 0.2661 & 0.3607 & -0.0733 & 0.068 \\
$\mathbf{H}(16)$ & $4 e$ & 0.2016 & 0.2391 & -0.1730 & 0.062 \\
$\mathbf{H}(18)$ & $4 e$ & 0.1602 & -0.4151 & -0.1779 & 0.068 \\
$\mathbf{H}(19)$ & $4 e$ & 0.0780 & -0.5640 & -0.0958 & 0.085 \\
$\mathbf{H}(20)$ & $4 e$ & -0.0348 & -0.3903 & -0.0715 & 0.095 \\
$\mathbf{H}(21)$ & $4 e$ & -0.0671 & -0.0688 & -0.1327 & 0.091 \\
$\mathbf{H}(22)$ & $4 e$ & 0.0133 & 0.0832 & -0.2159 & 0.071 \\
\hline
\end{tabular}

* e-mail: luciana.malpezzi@polimi.it 
Table 3. Atomic coordinates and displacement parameters (in $\AA^{2}$ ).

\begin{tabular}{|c|c|c|c|c|c|c|c|c|c|c|}
\hline Atom & Site & $x$ & $y$ & $z$ & $U_{11}$ & $U_{22}$ & $U_{33}$ & $U_{12}$ & $U_{13}$ & $U_{23}$ \\
\hline $\mathrm{Br}$ & $4 e$ & $0.39235(3)$ & $0.20478(9)$ & $0.01098(2)$ & $0.0904(4)$ & $0.1001(4)$ & $0.0440(3)$ & $0.0020(3)$ & $-0.0082(2)$ & $-0.0156(2)$ \\
\hline $\mathbf{N}$ & $4 e$ & $0.1418(2)$ & $-0.0653(6)$ & $-0.2603(2)$ & $0.041(2)$ & $0.049(2)$ & $0.052(2)$ & $0.008(1)$ & $0.003(1)$ & $0.013(2)$ \\
\hline$O(1)$ & $4 e$ & $0.2392(2)$ & $0.2566(4)$ & $-0.3132(2)$ & $0.062(2)$ & $0.042(2)$ & $0.050(2)$ & $0.008(1)$ & $0.002(1)$ & $0.007(1)$ \\
\hline$O(2)$ & $4 e$ & $0.2102(1)$ & $-0.2628(4)$ & $-0.4046(1)$ & $0.059(2)$ & $0.053(2)$ & $0.044(1)$ & $0.003(1)$ & $-0.007(1)$ & $0.001(1)$ \\
\hline$O(3)$ & $4 e$ & $0.1945(1)$ & $0.0972(5)$ & $-0.4362(1)$ & $0.060(2)$ & $0.062(2)$ & $0.052(1)$ & $0.003(1)$ & $-0.008(1)$ & $0.017(1)$ \\
\hline$C(1)$ & $4 e$ & $0.2165(2)$ & $-0.0435(6)$ & $-0.3946(2)$ & $0.036(2)$ & $0.056(2)$ & $0.041(2)$ & $0.001(2)$ & $0.004(1)$ & $0.008(2)$ \\
\hline$C(2)$ & $4 e$ & $0.2563(2)$ & $0.0260(5)$ & $-0.3249(2)$ & $0.043(2)$ & $0.041(2)$ & $0.041(2)$ & $0.004(2)$ & $0.004(1)$ & $0.005(1)$ \\
\hline$C(3)$ & $4 e$ & $0.2232(2)$ & $-0.1054(6)$ & $-0.2604(2)$ & $0.037(2)$ & $0.040(2)$ & $0.041(2)$ & $0.007(1)$ & $0.005(1)$ & $0.001(2)$ \\
\hline$C(4)$ & $4 e$ & $0.1776(2)$ & $-0.3309(7)$ & $-0.4717(2)$ & $0.077(3)$ & $0.065(3)$ & $0.049(2)$ & $-0.002(2)$ & $-0.008(2)$ & $-0.005(2)$ \\
\hline$C(5)$ & $4 e$ & $0.3420(2)$ & $-0.0088(5)$ & $-0.3311(2)$ & $0.046(2)$ & $0.044(2)$ & $0.030(2)$ & $-0.004(2)$ & $0.003(1)$ & $0.006(1)$ \\
\hline$C(6)$ & $4 e$ & $0.3909(2)$ & $0.1596(6)$ & $-0.3097(2)$ & $0.054(2)$ & $0.052(2)$ & $0.039(2)$ & $0.001(2)$ & $0.004(2)$ & $-0.004(2)$ \\
\hline$C(7)$ & $4 e$ & $0.4686(2)$ & $0.1335(7)$ & $-0.3158(2)$ & $0.054(2)$ & $0.063(2)$ & $0.045(2)$ & $-0.015(2)$ & $-0.003(2)$ & $0.000(2)$ \\
\hline$C(8)$ & $4 e$ & $0.4984(2)$ & $-0.0606(7)$ & $-0.3439(2)$ & $0.044(2)$ & $0.070(3)$ & $0.053(2)$ & $-0.003(2)$ & $0.005(2)$ & $0.007(2)$ \\
\hline$C(9)$ & $4 e$ & $0.4508(2)$ & $-0.2302(7)$ & $-0.3643(2)$ & $0.044(2)$ & $0.058(2)$ & $0.077(3)$ & $0.004(2)$ & $0.007(2)$ & $-0.009(2)$ \\
\hline$C(12)$ & $4 e$ & $0.3222(2)$ & $-0.1648(6)$ & $-0.1641(2)$ & $0.046(2)$ & $0.047(2)$ & $0.042(2)$ & $0.012(2)$ & $0.004(2)$ & $-0.002(2)$ \\
\hline$C(13)$ & $4 e$ & $0.3609(2)$ & $-0.0959(7)$ & $-0.1033(2)$ & $0.048(2)$ & $0.066(2)$ & $0.041(2)$ & $0.014(2)$ & $-0.001(2)$ & $0.003(2)$ \\
\hline$C(14)$ & $4 e$ & $0.3400(2)$ & $0.1025(6)$ & $-0.0708(2)$ & $0.055(2)$ & $0.061(2)$ & $0.032(2)$ & $0.001(2)$ & $0.009(1)$ & $0.001(2)$ \\
\hline$C(15)$ & $4 e$ & $0.2805(2)$ & $0.2285(6)$ & $-0.0962(2)$ & $0.081(3)$ & $0.047(2)$ & $0.043(2)$ & $0.013(2)$ & $0.008(2)$ & $-0.005(2)$ \\
\hline$C(16)$ & $4 e$ & $0.2424(2)$ & $0.1554(6)$ & $-0.1561(2)$ & $0.059(2)$ & $0.048(2)$ & $0.048(2)$ & $0.017(2)$ & $0.001(2)$ & $0.001(2)$ \\
\hline$C(17)$ & $4 e$ & $0.0953(2)$ & $-0.1515(6)$ & $-0.2067(2)$ & $0.039(2)$ & $0.047(2)$ & $0.044(2)$ & $-0.002(2)$ & $-0.000(1)$ & $-0.005(2)$ \\
\hline$C(18)$ & $4 e$ & $0.1141(2)$ & $-0.3444(6)$ & $-0.1695(2)$ & $0.045(2)$ & $0.056(2)$ & $0.070(2)$ & $0.000(2)$ & $0.006(2)$ & $0.013(2)$ \\
\hline$C(19)$ & $4 e$ & $0.0650(2)$ & $-0.4326(8)$ & $-0.1199(2)$ & $0.065(3)$ & $0.075(3)$ & $0.071(3)$ & $-0.012(2)$ & $0.002(2)$ & $0.019(2)$ \\
\hline$C(20)$ & $4 e$ & $-0.0022(3)$ & $-0.3308(9)$ & $-0.1055(2)$ & $0.063(3)$ & $0.110(4)$ & $0.066(3)$ & $-0.019(3)$ & $0.018(2)$ & $0.010(3)$ \\
\hline$C(21)$ & $4 e$ & $-0.0211(2)$ & $-0.1389(9)$ & $-0.1419(2)$ & $0.045(2)$ & $0.106(4)$ & $0.077(3)$ & $0.005(2)$ & $0.018(2)$ & $-0.014(3)$ \\
\hline$C(22)$ & $4 e$ & $0.0269(2)$ & $-0.0479(7)$ & $-0.1920(2)$ & $0.043(2)$ & $0.069(3)$ & $0.065(2)$ & $0.010(2)$ & $0.004(2)$ & $-0.003(2)$ \\
\hline
\end{tabular}

\section{References}

1. Clerici, A.; Clerici, L.; Porta, O.: A tricomponent reaction promoted by a titanium trichloride/pyridine system. Diastereoselective synthesis of $\beta$ amino- $\alpha$-hydroxyesters. Tetrahedron Lett. 36 (1995) 5955-5958.

2. Altomare, A.; Burla, M. C.; Camalli, M.; Cascarano, G. L.; Giacovazzo, C.; Guagliardi, A.; Moliterni, A. G. G.; Polidori, P.; Spagna, R.: SIR97: a new tool for crystal structure determination and refinement. J. Appl. Crystallogr. 32 (1999) 115-119.
3. Sheldrick. G. M.: SHELXL-97. Program for the Refinement of Crystal Structures. University of Göttingen, Germany 1997.

4. Sheldrick, G. M.: SHELXTL/PC. Release 5.0. Siemens Analytical X-ray Instruments, Inc., Madison, Wisconsin, USA 1996. 